Supporting information for,

\title{
The Effect of Short Chain Polyethyleneglycols on the Hydration Structure and Dynamics around Human Serum Albumin
}

\author{
Nirnay Samanta ${ }^{1, \dagger}$, Trung Quan Luong ${ }^{1, \star}$, Debasish Das Mahanta ${ }^{\dagger}$, \\ Rajib Kumar Mitra ${ }^{\dagger, *}$ and Martina Havenith ${ }^{\text {*** }}$
}

\footnotetext{
${ }^{\dagger}$ Department of Chemical, Biological and Macromolecular Sciences, S.N. Bose National Centre for Basic Sciences, Block JD, Sector III, Salt Lake, Kolkata 700098, India

* Department of Physical Chemistry II, Ruhr-University Bochum, 44780 Bochum, Germany 1. Contributed equally to this work.

*E-mail: martina.havenith@rub.de; rajib@bose.res.in
} 


\section{Materials and Methods:}

\section{Fluorescence Anisotropy measurements:}

For the steady state anisotropy measurement emission polarization is adjusted to be parallel or perpendicular to that of the excitation and anisotropy is obtained by the following equation

$$
r=\frac{I_{\|}-I_{\perp}}{I_{\|}+2 I_{\perp}}
$$

where $I_{\|}$and $I_{\perp}$ are the intensities measured with a linear polarizer for emission (the analyzer) parallel and perpendicular, respectively, to the electric vector of linearly polarized incident light.

TTDS measurements: TTDS measurements were carried out in a commercial $\mathrm{THz}$ spectrophotometer (TERA K8, Menlo System). A $780 \mathrm{~nm}$ Er doped fiber laser having pulse width of $<100 \mathrm{fs}$ and a repetition rate of $100 \mathrm{MHz}$ excites a $\mathrm{THz}$ emitter antenna producing a $\mathrm{THz}$ radiation having a bandwidth up to $3.0 \mathrm{THz}(>60 \mathrm{~dB})$. This $\mathrm{THz}$ radiation is then focused on sample and the transmitted $\mathrm{THz}$ radiation is further focused on a $\mathrm{THz}$ detector antenna which is gated by the probe laser beam. Both the $\mathrm{THz}$ antennas are gold dipoles with a dipole gap of $5 \mu \mathrm{m}$ deposited on LT-GaAs substrate. To avoid water vapour absorption, all the measurements were carried out in dry nitrogen atmosphere with a controlled humidity of $<10 \%$ at $293 \mathrm{~K}$ using a liquid cell (Bruker, model A 145) using z-cut quartz windows and Teflon spacer of $100 \mu \mathrm{m}$ thickness. Samples were reloaded for four times in the sample cell and nine full scans were averaged together to minimize the error in the results. By varying the time delay between the probe and the pump beam the amplitude and phase of the THz electric field are measured as a function of time. Frequency dependent power and phase of the transmitted pulse are obtained using Fourier analysis of the measured electric field amplitude $\mathrm{E}_{\mathrm{THz}}(\mathrm{t})$. Subsequently, The frequency dependent real $(\varepsilon)$ and imaginary $\left(\varepsilon^{\prime}\right)$ dielectric constants of the samples are extracted as, $\varepsilon^{\prime}=n^{2}(v)-k^{2}(v)$ and $\varepsilon^{\prime \prime}(v)=2 n(v) k(v)$ where the complex refractive index is given as, $\tilde{n}(v)=n(v)-i k(v)$.

\section{Debye dielectric relaxation model:}

According to the Debye model, the complex frequency dependent dielectric response can be described as:

$$
\tilde{\varepsilon}(v)=\varepsilon_{\infty}+\sum_{j=1}^{m} \frac{\varepsilon_{j}-\varepsilon_{j+1}}{1+i 2 \pi v \tau_{j}}
$$


where, $\tau_{j}$ is the relaxation time for the $j$-th relaxation mode, $\varepsilon_{1}$ is the static dielectric constant, $\varepsilon_{j}$ are the dielectric constants for different relaxation processes, $\varepsilon_{\infty}$ is the extrapolated dielectric constant at a very high frequency, and $m$ describes the number of relaxation modes. The magnitude of induced polarization is given by the dispersion amplitude, $S_{j}=\varepsilon_{j}-\varepsilon_{j+1}$. In our earlier studies we fitted the relaxation data of water in this frequency range using three different time scales. Keeping in mind that a considerable fraction of water molecules in these concentrated solutions move rather slowly, we model the DR by introducing an additional Debye mode; the following equation describes the frequency-dependent complex permittivity, $\tilde{\varepsilon}(\omega)$ :

$$
\tilde{\varepsilon}(\omega)=\varepsilon_{\infty}+\frac{S_{1}}{1+i \omega \tau_{1}}+\frac{S_{2}}{1+i \omega \tau_{2}}+\frac{S_{3}}{1+i \omega \tau_{3}}
$$

where $\mathrm{S}_{\mathrm{i}}$ is the relaxation strength of the $\mathrm{i}$-th mode and $\omega$ is the angular frequency. 


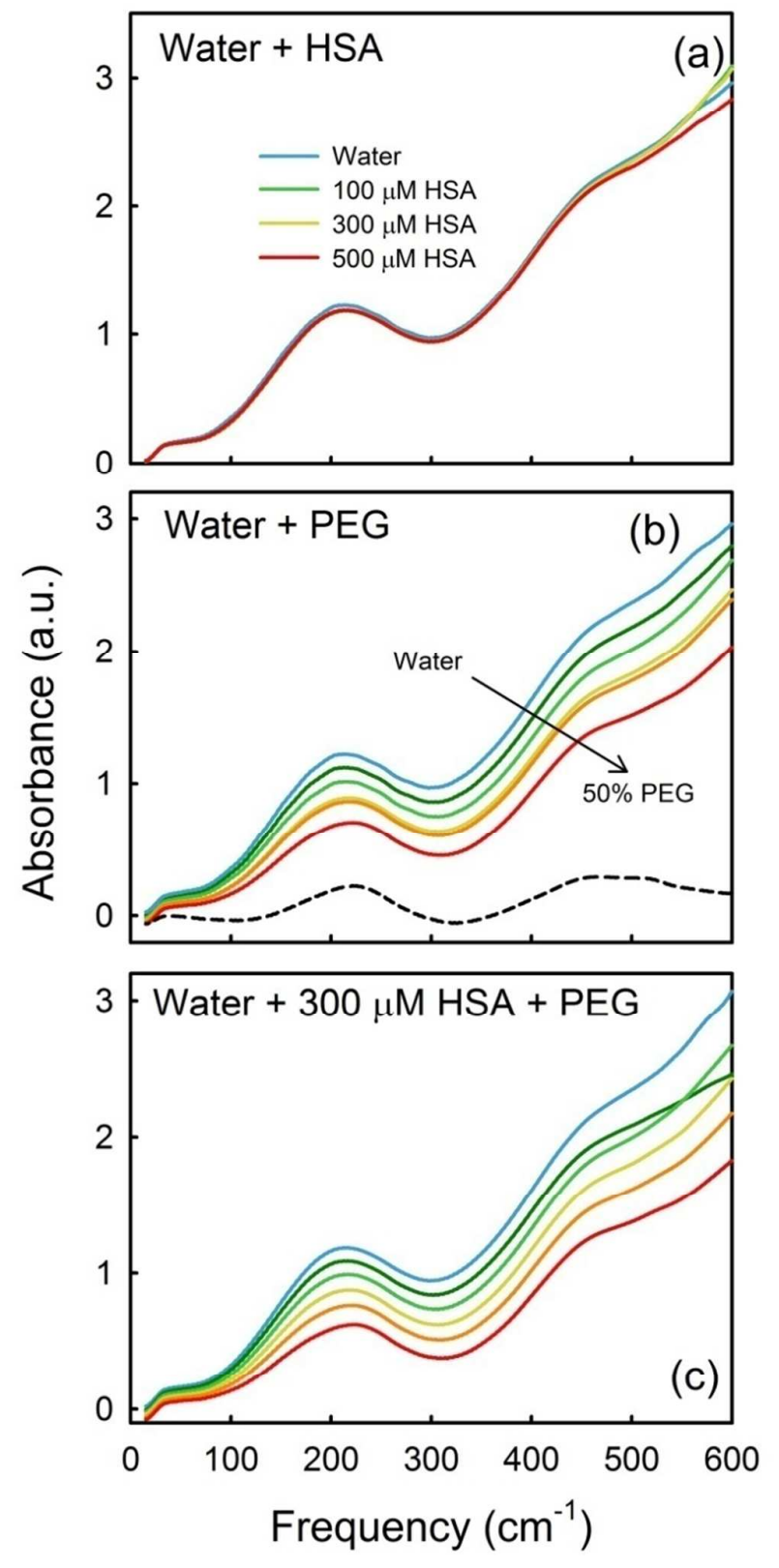

Figure S1. (a) FIR-FTIR of water and HSA at different concentrations. (b) FIR-FTIR of PEG 400 at different concentration. The absorption spectrum of pure PEG 400 is shown as a broken line. (c) FIR-FTIR of HSA in presence of different concentrations of PEG 400. 


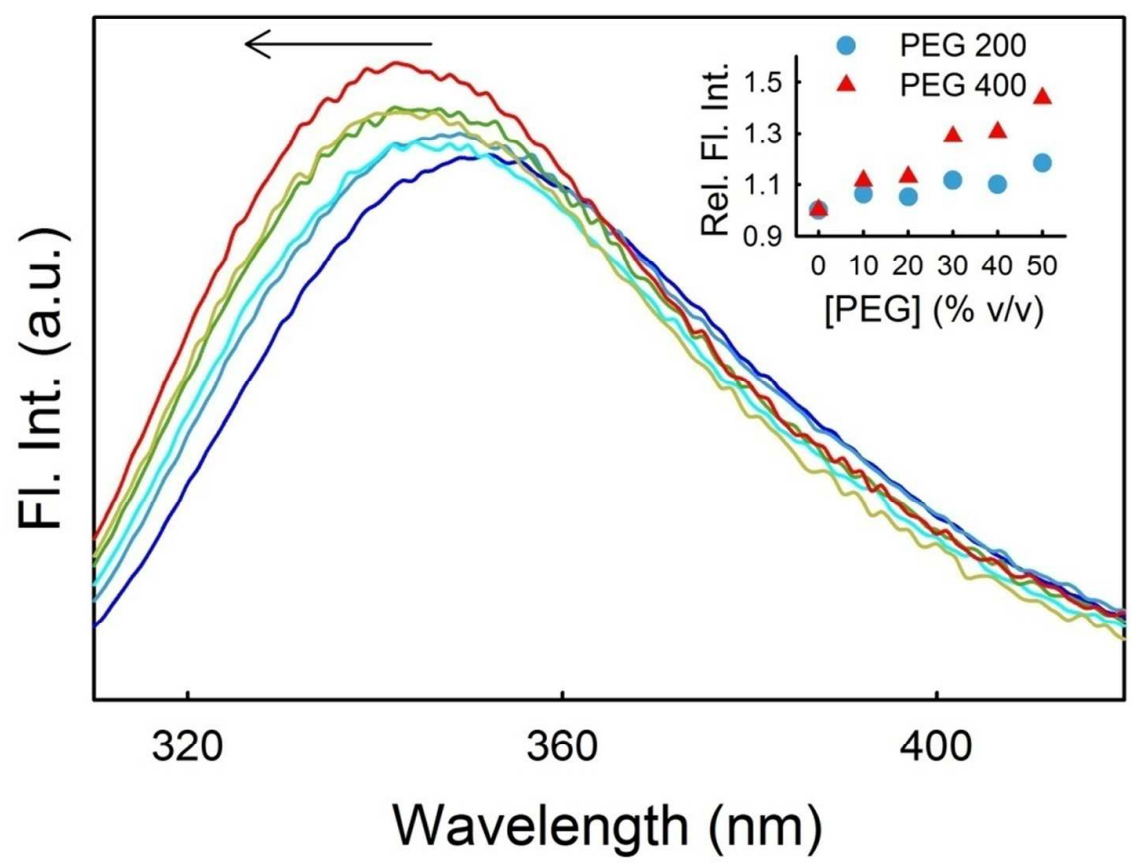

Figure S2. Emission profile of Trp214 of HSA in buffer and in presence of PEG 400 at different concentrations. The arrow indicates increasing PEG concentration. The relative fluorescence intensity of Trp214 in HSA has been plotted in the inset as a function of PEG 200 and PEG 400 concentrations. 


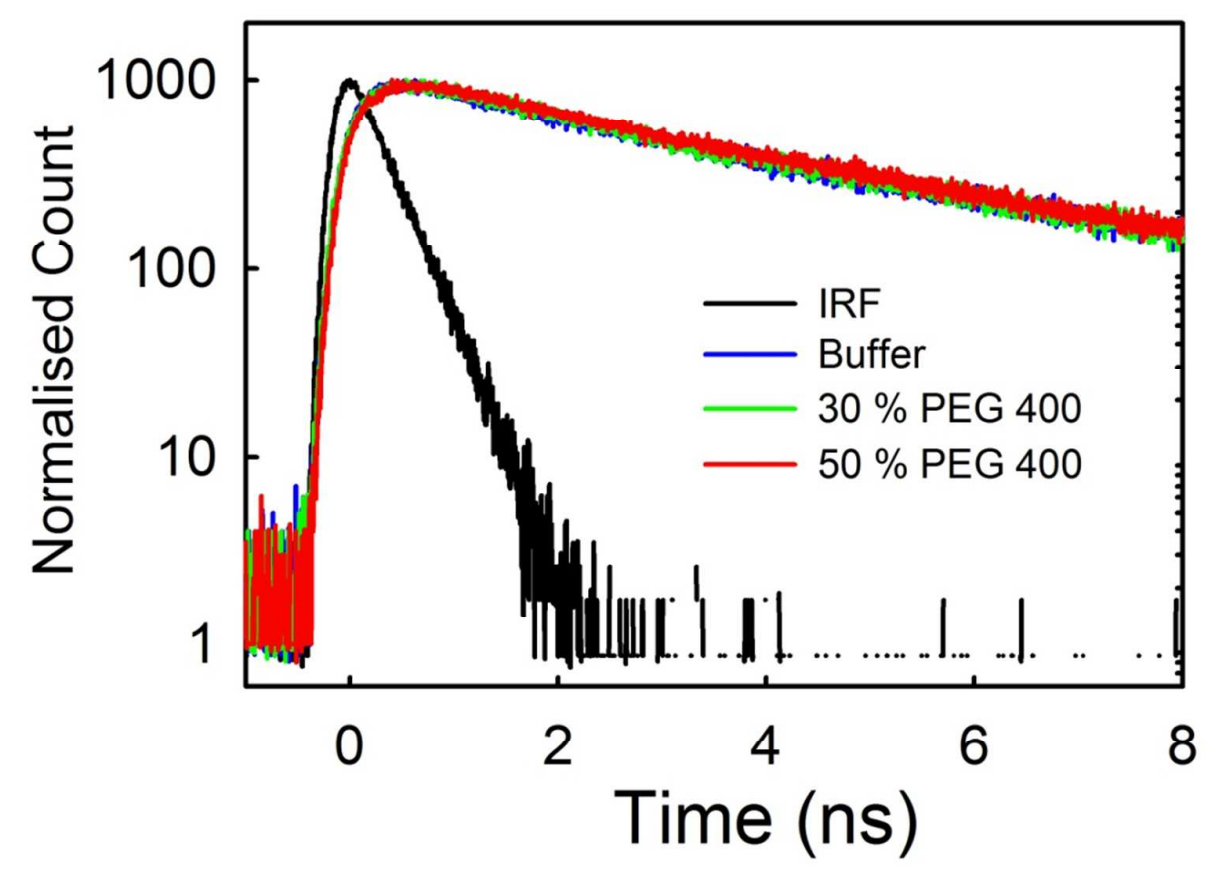

Figure S3. Temporal decay of Trp214 in HSA in absence and in presence of PEG 400. 


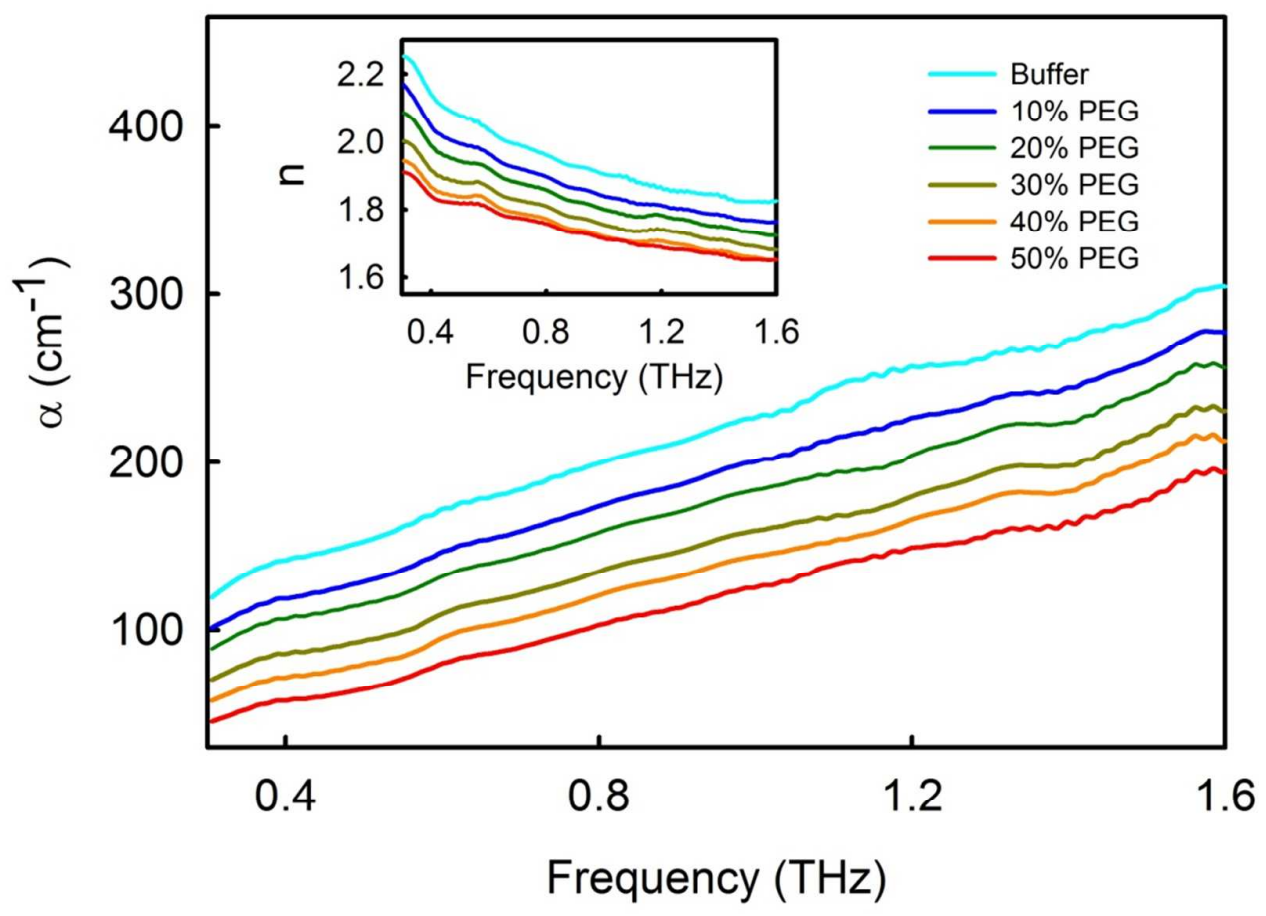

Figure S4. Frequency dependent absorption coefficient, $\alpha$ and refractive index, $\mathrm{n}$ of buffer and PEG 400 solutions at different concentrations. 
Table S1. Debye relaxation fitting parameters of aqueous solutions of PEG 400 at different concentrations

\begin{tabular}{|c|c|c|c|c|c|c|c|}
\hline $\begin{array}{c}{[\text { PEG] }} \\
(\mathbf{\%} \mathbf{v} / \mathbf{v})\end{array}$ & $\boldsymbol{\varepsilon}_{\infty}$ & ${ }^{(\mathbf{a})} \boldsymbol{\varepsilon}_{\mathbf{s}}$ & $\boldsymbol{\varepsilon}_{\mathbf{2}}$ & $\boldsymbol{\varepsilon}_{\mathbf{3}}$ & $\begin{array}{c}\tau_{\mathbf{1}} \\
\mathbf{( p s )}\end{array}$ & $\begin{array}{c}\tau_{2} \\
(\mathbf{f s})\end{array}$ & $\begin{array}{c}\tau_{3} \\
(\mathbf{f s})\end{array}$ \\
\hline 0 & $2.13 \pm 0.03$ & 75.13 & $3.92 \pm 0.06$ & $3.49 \pm 0.10$ & $8.86 \pm 0.2$ & $222 \pm 65$ & 84 \\
\hline 10 & $2.01 \pm 0.03$ & 71.73 & $3.81 \pm 0.06$ & $3.31 \pm 0.07$ & $11.08 \pm 0.2$ & $239 \pm 54$ & 84 \\
\hline 20 & $1.99 \pm 0.02$ & 65.67 & $3.69 \pm 0.06$ & $3.20 \pm 0.08$ & $11.85 \pm 0.2$ & $239 \pm 57$ & 84 \\
\hline 30 & $1.90 \pm 0.03$ & 58.76 & $3.57 \pm 0.06$ & $3.07 \pm 0.08$ & $14.58 \pm 0.4$ & $237 \pm 54$ & 83 \\
\hline 40 & $1.82 \pm 0.03$ & 51.48 & $3.48 \pm 0.06$ & $2.96 \pm 0.08$ & $16.70 \pm 0.5$ & $234 \pm 54$ & 83 \\
\hline 50 & $1.86 \pm 0.02$ & 44.65 & $3.43 \pm 0.03$ & $2.90 \pm 0.08$ & $19.59 \pm 0.6$ & $210 \pm 34$ & 84 \\
\hline
\end{tabular}

(a) Values are taken from Ind. J. Pure Appl. Phys. (2007) 45, 476-481. 


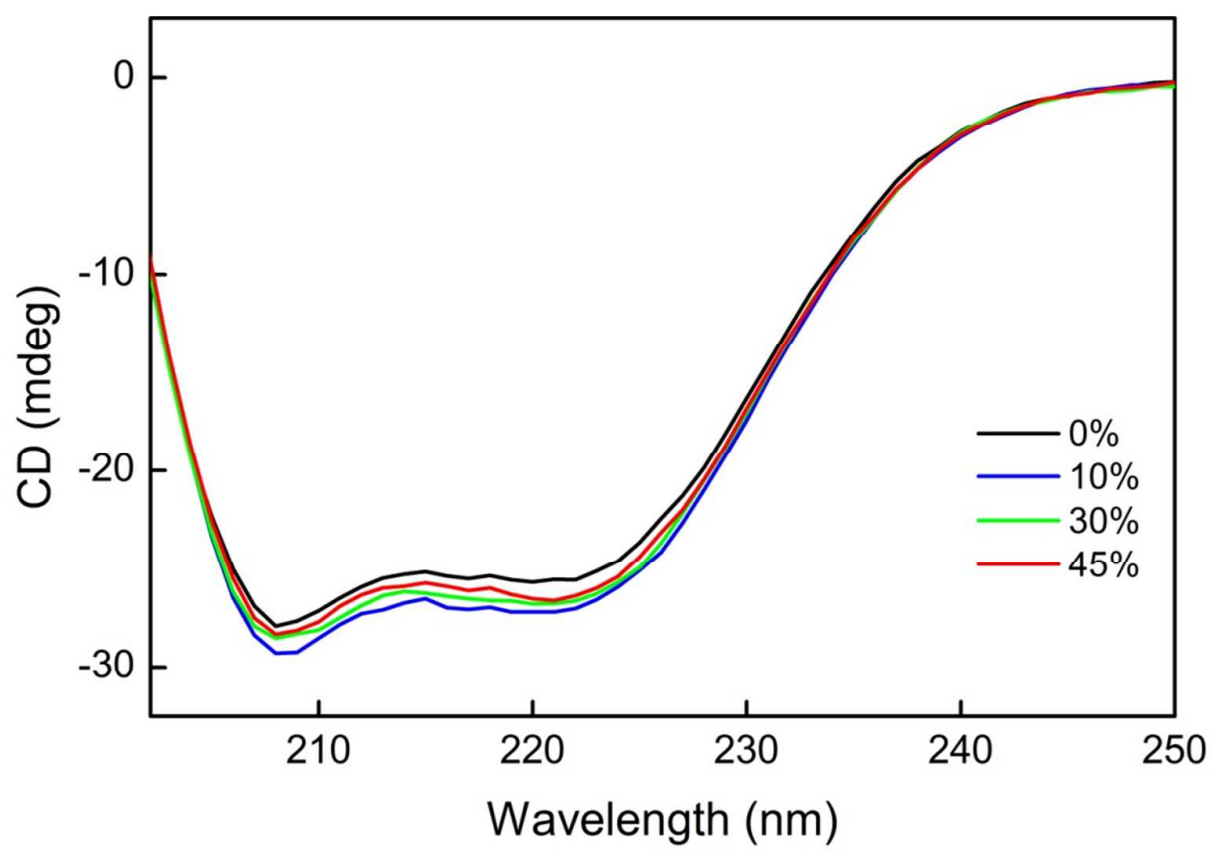

Figure S5. Far-UV CD spectra of HSA at different concentrations of Sucrose. 


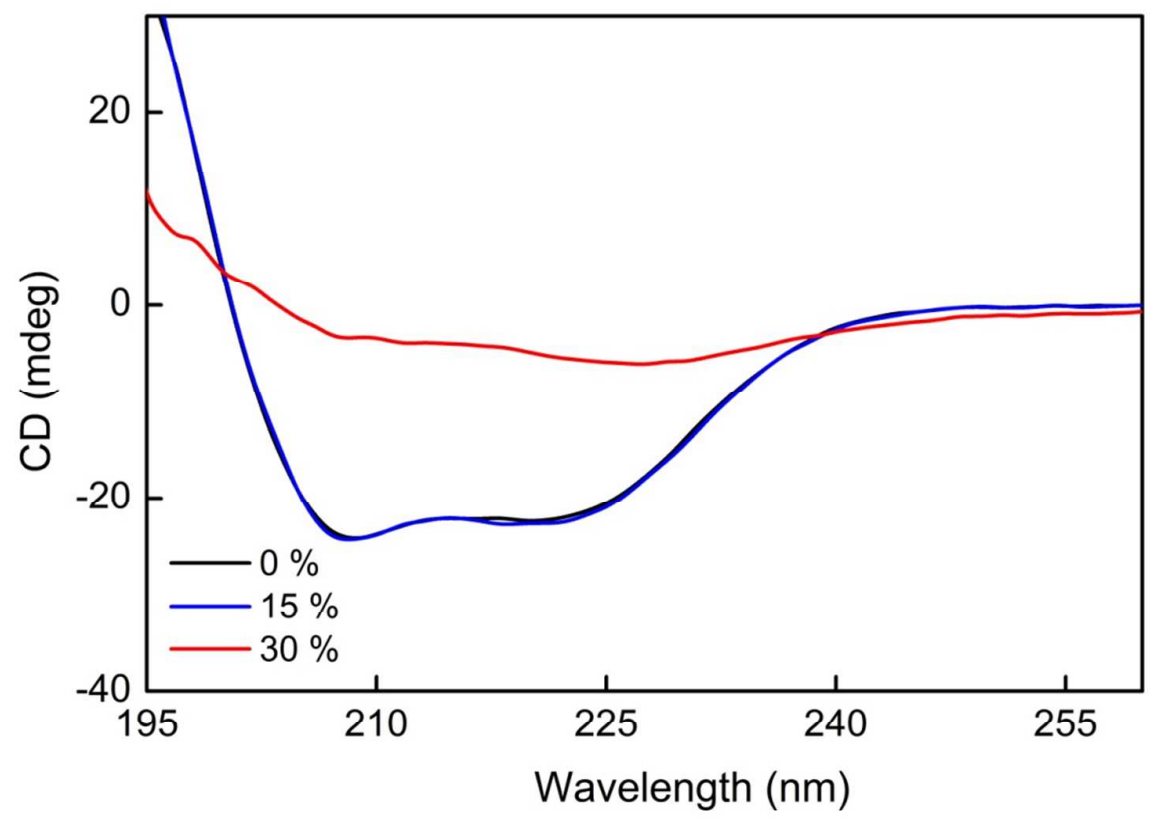

Figure S6. Far-UV CD spectra of HSA at different concentrations of PEG 10000. 